\title{
Commentary
}

\section{Making Public Health Nutrition relevant to evidence-based action}

\author{
Eric Brunner ${ }^{1, *}$, Mike Rayner ${ }^{2}$, Margaret Thorogood $^{3}$, Barrie Margetts ${ }^{4}$, Lee Hooper ${ }^{5}$, \\ Carolyn Summerbell ${ }^{6}$, Elizabeth Dowler ${ }^{7}$, Gillian Hewitt $^{8}$, Aileen Robertson ${ }^{9}$ and \\ Martin Wiseman ${ }^{10}$ \\ 'International Centre for Health and Society, Department of Epidemiology and Public Health, University College \\ London, 1-19 Torrington Place, London WC1E 6BT, UK: ${ }^{2}$ British Heart Foundation Health Promotion Research \\ Group, Department of Public Health, University of Oxford, Oxford OX3 7LF, UK: ${ }^{3}$ Department of Public Health \& \\ Policy, London School of Hygiene and Tropical Medicine, London WC1E 7HT, UK: ${ }^{4}$ Public Health Nutrition, Institute \\ of Human Nutrition, University of Southampton, Southampton SO 16 6YD, UK: ${ }^{5}$ Manchester Dental and Education \\ Centre (MANDEC), University Dental Hospital of Manchester, Manchester M15 6FH, UK: 'S School of Health, \\ University of Teesside, Middlesbrough, Cleveland, TS1 3BA, UK: ${ }^{7}$ Department of Sociology, University of Warwick, \\ Coventry CV4 7AL, UK: ${ }^{8}$ London School of Hygiene and Tropical Medicine, London WC1E 7HT, UK: ${ }^{9}$ Acting \\ Regional Advisor for Nutrition, WHO Regional Office for Europe, Copenhagen, Denmark: ${ }^{10}$ World Cancer \\ Research Fund, London WIN 1DA, UK
}

Public health nutrition enjoyed many breakthroughs in the 20 th century - from the discovery of vitamins and the metabolic roles of some 60 macro- and micronutrients, to the effects of maternal and childhood diet on health over the life course. Moreover, the food shortages in the UK that were experienced during World War II gave the first opportunity to show that nutritional science could make a valuable contribution to public policy. However, public health nutrition is now facing the challenge of deriving recommendations based on systematically evaluated evidence; that is, the new concept of evidence-based nutrition. This challenge was foreseen by John Garrow almost 10 years ago in his lucid editorial proposing the formation of meta-analysis 'clubs'.

The 2000 Eurodiet Conference was the culmination of a two-year EU-wide scientific process funded by the European Commission, which ended with a set of population dietary goals ${ }^{2}$. These recommendations were intended to provide the basis for future EU-wide nutrition action. However, they have only been partly adopted. A Council Resolution on health and nutrition has cited increased fruit and vegetable consumption and increased breast-feeding as possible priorities for European nutrition policy (Council of Ministers' resolution, 14 December 2000), but other recommendations, such as those on total fat and saturated fatty acids, iron, sodium, potassium and calcium, have yet to reach even this tentative recognition. One of the reasons for this hesitancy can be found in the closing speeches of the Eurodiet conference, when Matti Rajala, from the Directorate General, asked for nutritional evidence to be assembled in a more transparent and accessible way. Rajala observed that the conference had been debating what evidence should be cited, and he doubted that all of the relevant studies had been considered. He pointed to the absence of systematically collated evidence to support recommendations, and called for less reliance on consensus documents, and more reliance on systematic accumulation, synthesis and presentation of evidence. In summary, he was calling for more evidence-based nutrition.

A definition of evidence-based nutrition can be derived very simply from one well-known definition of evidencebased public health ${ }^{3}$. Evidence-based nutrition is the application of the best available systematically assembled evidence in setting nutrition policy and practice. The need for new methods of dealing with published data is clear from Fig. 1. The amount of published work is increasing rapidly, and is quite beyond any one person to handle rationally. Systematic methods to synthesise information are available and there is an urgent need to use them more widely.

The methods are already being applied in nutrition ${ }^{4-7}$. The Committee on Medical Aspects of Food Policy's (COMA) recent review of nutrition and cancer ${ }^{8}$ is the first government report to use this approach to summarising evidence. Well-conducted and clearly reported systematic reviews are invaluable in clarifying complicated or contentious issues, and sometimes the results challenge existing wisdom. A recent pooled analysis of eight prospective studies of breast cancer and fruit and vegetable intake, involving more than 350000 women, did not find a link between the two 9 . This is an important result, given the 'probable' protective effect reported in a previous high-profile review ${ }^{10}$.

Evidence-based nutrition has found a place in the Cochrane Library. The most recent edition of the Cochrane 


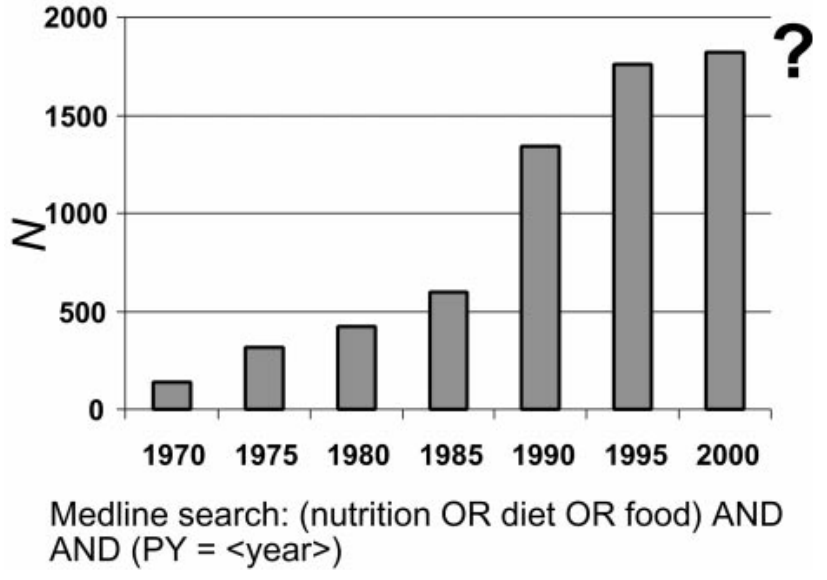

Fig. 1 Medline citations of papers including nutritional items by publication year

Library $^{11}$ contains 60 complete reviews involving one or more nutritional interventions. However, the model of a Cochrane Review, which is typically a metaanalysis of randomised controlled trials, does not translate comfortably into the field of nutrition. Identifying effective interventions in nutrition often requires studying diet over longer periods and in larger populations than would ever be practical in a randomised controlled trial, even supposing participants could be found who would agree to long-term engineering of their dietary intake.

Moreover, the scope of evidence-based nutrition is far wider than randomised trials. To construct effective and relevant policy recommendations, evidence is certainly needed on the relationship between diet and health but evidence is also needed on the acceptability of foods, on efficiency of food distribution, on the psychological or social issues that affect food preference, and so on. The evidence that will be used will come from observational studies, from economic modelling exercises, from sociological studies and from many other sources, as well as from randomised controlled trials. What is important is that evidence is assembled systematically, with transparent inclusion and exclusion criteria, with attention paid to the methodological quality of the work, and without prior assumptions about the findings being allowed to influence what evidence is considered. The key principles of systematic reviews are summarised in the Appendix.

The COMA working group ${ }^{8}$ reviewing links between nutrition and cancer drew heavily on earlier work of the International Agency for Research on Cancer (IARC) ${ }^{12}$. The vast majority of studies reviewed were either casecontrol or cohort studies; there were few randomised controlled trials. When all available literature was compiled, deliberations about drawing conclusions from the data were based upon: the type of epidemiological study; consistency of results between studies; the quality of studies reviewed (using a scoring system ${ }^{13}$ ); a general tendency for the results of all studies to be in the same direction; the size of the relative risk; whether there was a graded response; evidence of an effect from randomised controlled trials; evidence of the exposure preceding the effect; and whether there was any evidence of a plausible mechanism. In the end the conclusions reached were still matters of judgement, but at least the evidence had been laid out in a clear, open and transparent way, and all the evidence was judged in the same way.

A commitment to evidence-based nutrition will require shifting the focus of debate from sterile dissent about what evidence is relevant to working together to explain heterogeneity in the evidence. The 'latest study supports my view' approach to knowledge is seriously weakened by systematic review principles.

Converting evidence to policy is a difficult process, and this is particularly true of setting population nutrition goals, as the Eurodiet participants found. The continuing debates about the efficacy and effectiveness of recommendations on dietary fat and fat fractions, salt, extrinsic sugar and various micronutrients will only move forward productively if an evidence-based approach is adopted. However, evidence-based nutrition is not a panacea. Vested interests inevitably influence debates in nutrition, and will continue to do so. Furthermore, data are not available in equivalent quantity or quality for all types of decisions. There is far more evidence related to interventions at individual level than at population level, and this imbalance may distort policy-making. (This, once again, highlights the importance of a broad-based approach to the type of evidence included in systematic reviews.)

\section{Moving towards evidence-based nutrition}

How could evidence-based nutrition be promoted? Nutritionists need systematic review skills, particularly in critical appraisal, database searching and quantitative methods. Departments of nutrition and others with a research interest in the nutrition field must alter the emphasis in their undergraduate and postgraduate courses, and add data synthesis to their research portfolios, for example through doctoral projects. We propose that a European network be established that brings nutritionists together with epidemiologists, biostatisticians and other relevant specialists. A series of international workshops on evidence-based nutrition could be a major contribution of the proposed network.

There are many models for a collaborative review network in nutrition. Defining its tasks and methods will require discussion, but the potential outputs of such a network are exciting. A library of reviews in a standard format on CD-ROM and the Internet would be central. Questions of methodology, aetiology, efficacy and effectiveness are all suitable for systematic review. 


\section{Conclusion}

Public Health Nutrition will be revitalised by systematic, evidence-based methods that will deliver clarity of evidence and effective policy recommendations, thereby producing public health gains. Public Health Nutrition can have the impact it deserves, but only if the evidence is brought together and applied to the solution of real problems.

\section{Statement of interest}

EB and LH are unpaid editors in the Cochrane Heart Group.

\section{References}

1 Garrow JS. Would clinical nutrition benefit from metaanalyses and trials registers. Eur. J. Clin. Nutr. 1992; 46: $843-5$.

2 Kafatos AG, Codrington CA, eds. Eurodiet Reports and Proceedings [special issue]. Public Health Nutr. 2001; 4(2A): $265-436$.

3 Last JM, ed. A Dictionary of Epidemiology. 3rd ed. New York: Oxford University Press 1995.

4 Brunner EJ, White IR, Thorogood M, Bristow A, Curle D, Marmot MG. Can dietary interventions change diet and cardiovascular risk factors? A meta-analysis of randomized controlled trials. Am. J. Public Health 1997; 87: 1415-22.

5 Cutler JA, Follmann D, Allender PS. Randomised trials of sodium reduction: an overview. Am. J. Clin. Nutr. 1997 65: $643 S-51 S$

6 Hooper L, Summerbell CD, Higgins JPT, Thompson RL, Capps NE, Davey Smith G, Riemersma RA, Ebrahim S. Dietary fat intake and prevention of cardiovascular disease: systematic review. Br. Med. J. 2001; 322: 757-63.

7 Mensink RP, Katan MB. Effect of dietary fatty acids on serum lipids and lipoproteins: a meta-analysis of 27 Trials. Art. Thromb. 1992; 12: 911-919.

8 Committee on Medical Aspects of Food Policy. Nutritional
Aspects of the Development of Cancer. London: The Stationery Office, 1998.

9 Smith-Warner SA, Spiegelman D, Yaun SS, et al. Intake of fruits and vegetables and risk of breast cancer: a pooled analysis of cohort studies. JAMA 2001 285: 769-76.

10 World Cancer Research Fund. Food, Nutrition and the Prevention of Cancer: A Global Perspective. Washington, DC: American Institute for Cancer Research, 1997.

11 Cochrane Library. Oxford: Update Software.

12 International Agency for Research on Cancer (IARC). Some Naturally Occurring Substances: Food Items and Constituents, Heterocyclic Amines and Mycotoxins. IARC Monographs on the Evaluation of Carcinogenic Risks of Chemicals to Humans Lyon: IARC, 1993; 56.

13 Margetts BM, Thompson RL, Key T, Duffy S, Nelson M, Bingham S, Wiseman M. Development of a scoring system to judge the scientific quality of information from case-control and cohort studies of nutrition and cancer. Nutr. Cancer 1995; 24: 231-9.

\section{Appendix - Principles of systematic reviews}

\section{TRANSPARENCY AND QUALITY}

- unambiguously stated research question

- explicit inclusion and exclusion criteria, independent of study findings

- reproducible and extensive search strategy

- peer-reviewed protocol

\section{ACCESSIBLITY}

- promotion of access and wide dissemination

\section{CONTINUITY}

- maintained and updated reviews

RELEVANCE

- subjects of public health importance

- duplication of reviews avoided by co-ordination 